\title{
(1) RCEC
}

\section{ASSOCIAÇÃO DA CARBOXITERAPIA E INTRADERMOTERAPIA NO TRATAMENTO DA LIPODISTROFIA LOCALIZADA RELATO DE CASO}

\author{
Thuanny Farina'; Sara Petroni²; Lidiane Pereira da Rocha ${ }^{3, A}$
}

${ }^{1}$ Biomédica -UniFMU; Pós graduada em Estética e Cosmetologia - AVM.

Biomédica -UniFMU; Pós graduada em Estética e Cosmetologia - Unyleya.

${ }^{3}$ Esteticista, Especialista em pré e pós procedimentos Médicos - UAM, Mestre em Biofotônica - Universidade Nove de Julho (UNINOVE) - São Paulo - SP.

\section{RESUMO}

\begin{abstract}
A Lipodistrofia localizada conhecida popularmente como gordura localizada, pode ser definida como uma distribuição regional de gordura classificada em ginóide (quando a deposição excessiva está localizada em quadril e coxas) e androide (quando a deposição excessiva é na área abdominal). 0 estudo de caso foi realizado em uma mulher de 34 anos com queixa de gordura localizada na região abdominal. Para essa pesquisa foram realizadas 10 aplicações com intervalos semanais do protocolo combinando Carboxiterapia e Intradermoterapia Tradicional. Os resultados clínicos evidenciaram redução abdominal significativa avaliada através de perimetria e avaliações fotográficas.
\end{abstract}

Palavras Chaves: Lipodistrofia localizada, Abdominal, Carboxiterapia, Intradermoterapia.

\section{ABSTRACT}

Localized lipodystrophy, popularly known as localized fat, can be defined as a regional distribution of fat classified into gynoid (when excessive deposition is located in the hips and thighs) and android (when excessive deposition is in the abdominal area). The case study was carried out in a 34-year-old woman complaining of localized fat in the abdominal region. For this research, 10 applications were performed at weekly intervals of the protocol combining Carboxytherapy and Traditional Intradermotherapy. Clinical results showed significant abdominal reduction assessed through perimetry and photographic evaluations.

Keywords: Localized lipodystrophy, Abdominal, Carboxytherapy, Intradermotherapy.

DOI: https://doi.org/10.48051/rcec.v1i2.36 Artigo recebido em 10 de dezembro de 2021; aceito em 18 de dezembro 2021; publicado em 22 de dezembro de 2021 na Revista Científica de Estética e Cosmetologia, disponível online em http://rcec.healthsciences.com.br. Todos os autores contribuíram igualmente com o artigo. Os autores declaram não haver conflito de interesse. Este é um artigo de acesso aberto sob a licença CC - BY: http://creativecommons.org/licenses/by/4.0 


\section{INTRODUÇÃO}

Entre as diversas técnicas existentes para a redução da lipodistrofia localizada ou gordura localizada, como é conhecida popularmente, os métodos minimamente invasivos são muito requisitados principalmente pelo público feminino.

O tecido adiposo é uma forma especializada do tecido conjuntivo, formado por células chamadas adipócitos. O excedente das reservas nutricionais resultante de um desequilíbrio entre a ingestão de nutrientes e a necessidade diária é estocado no interior dos adipócitos sob a forma de triglicerídeos [1].

A principal função do tecido subcutâneo ou adiposo é armazenar ácidos graxos sob a forma de triglicerídeos, sendo então o maior reservatório energético do corpo humano [2].

$\mathrm{O}$ aumento do tecido adiposo promove alterações estéticas, muitas vezes dietas alimentares e atividade física não são suficientes para reduzir a lipodistrofia localizada em determinadas regiões, principalmente na região abdominal. Alguns tratamentos estéticos são indicados para o tratamento da gordura localizada, por suas ações facilitadoras da lipólise (hidrólise ou quebra dos triglicerídeos). A carboxiterapia consiste na infusão controlada de gás carbônico medicinal na pele e no tecido adiposo, a técnica utiliza um gás atóxico, estéril e não embólico, componente presente naturalmente no organismo, por isso, além de não deixar resíduos também auxilia nos processos relativos ao aumento do metabolismo celular[3].

Os efeitos fisiológicos previstos após a aplicação da carboxiterapia são: vasodilatação arteriovenosa, aumento do volume e da velocidade do fluxo sanguíneo local, aumento da drenagem sanguínea e linfática, ativação da microcirculação local melhora da nutrição celular, auxilio na eliminação de toxinas, ação lipolítica e redução do processo inflamatório. Graças a essas reações fisiológicas, o uso da carboxiterapia promove: melhoria na elasticidade e tônus tecidual, diminuição de edema, favorece a cicatrização e atua na redução de medidas e remodelagem corporal. Seu mecanismo de ação, causa fratura direta na membrana adipocitária e alteração na curva de dissociação da hemoglobina com o oxigênio, promovendo assim uma verdadeira ação lipolítica oxidativa[4, 5]

Após avaliações histológicas nas regiões tratadas, foi observado fratura da membrana do adipócito, aumento da espessura da pele e preservação total do tecido conectivo, inclusive de estruturas vasculares e nervosas, conclui-se então, que o gás carbônico não causa efeitos adversos ou secundários no tecido conectivo e estrutura nervosa, podendo ser considerado como sintomas secundários: dor no local da aplicação, pequenas equimoses ou hematomas, aumento da temperatura local devido à velocidade do fluxo limiar do paciente [5].

A mesoterapia ou intradermoterapia é um procedimento minimamente invasivo introduzido por Pistor, em 1958. Consiste na aplicação de substâncias farmacológicas muito diluídas diretamente na região a ser tratada, através de injeções intradérmicas. Pistor, em 1976, resumiu a técnica com as seguintes palavras: "Pouco, poucas vezes, e no local adequado". O próprio fundador da intradermoterapia reconheceu que tais recomendações eram empíricas e baseadas em sua experiência clínica pessoal. Ele afirmou ter percebido que, enquanto doses maiores não faziam diferença para o resultado clínico, múltiplas puncturas pareciam melhores que poucas injeções[6].

As injeções são capazes de estimular o tecido que recebe os ativos tanto pela ação da punção quanto pela ação dos fármacos [7].

Atualmente diversos profissionais da área de saúde utilizam a intradermoterapia para tratamentos estéticos de lipodistrofia localizada, fibro edema gelóide (celulite), disfunções capilares, entre outros [8].

A procura por tratamentos estéticos que promovam a redução de medidas e circunferência abdominal é alta, porém, a literatura é escassa, principalmente sobre intradermoterapia. Poucos estudos sobre métodos, eficácia e associações são encontrados, apesar de ser uma técnica muito utilizada em clínicas de estética com apresentação de resultados clínicos satisfatórios. Realizamos esse relato de caso para verificar a eficácia da combinação da Carboxiterapia e Intradermoterapia tradicional no tratamento da lipodistrofia localizada.

\section{MATERIAIS E MÉTODOS}

Trata-se de um relato de caso realizado na clínica Dra. Sara Petroni, na cidade de São Paulo. A paciente foi informada a respeito da pesquisa, procedimentos, riscos e benefícios e ao estar de acordo, assinou o Termo de Consentimento Livre e Esclarecido (TCLE), ficha de anamnese e Termo de Usufruto de Imagem.

O estudo foi realizado em uma paciente do sexo feminino, de 34 anos que apresentava lipodistrofia localizada na região abdominal. $O$ recrutamento da participante foi feito através de divulgação na clínica.

A participante foi submetida a 10 intervenções de carboxiterapia associada a intradermoterapia tradicional na região abdominal. Foram realizadas aplicações de $80 \mathrm{ml}$ de gás carbônico medicinal por ponto de aplicação (aproximadamente 12 pontos distribuídos no abdômen, com distância de $3 \mathrm{~cm}$ a $5 \mathrm{~cm}$ entre eles) com velocidade de $150 \mathrm{mlCO} 2 / \mathrm{min}$.

Imediatamente após, foi realizado o procedimento de intradermoterapia, com aplicações subcutâneas da mescla (princípios ativos), foi injetado $0,2 \mathrm{ml}$ por ponto de aplicação, sendo esses pontos distribuídos a cada $3 \mathrm{~cm}$ entre eles, em todo abdômen totalizando $10 \mathrm{ml}$ de mescla com seguintes ativos: Desoxicolato de sódio $1 \%$, cafeína $26 \mathrm{mg}$, silício $20 \mathrm{mg}$ e lidocaína $40 \mathrm{mg}$. 
FARINA, T. E COLABORADORES - REVISTA CIENTÍFICA DE ESTÉTICA E COSMETOLOGIA - V1 - N2

Tabela 1 - Parâmetros Carboxiterapia

\begin{tabular}{|c|c|c|c|}
\hline Pontos (região abdominal) & Distância entre pontos & Velocidade do gás & Quantidade de gás por ponto \\
\hline 12 & 3 a $5 \mathrm{~cm}$ & $150 \mathrm{ml} \mathrm{CO} / \mathrm{min}$ & $80 \mathrm{ml}$ \\
\hline
\end{tabular}

Tabela 2 - Ativos da Mescla para Intradermoterapia

\begin{tabular}{|c|c|}
\hline Princípios ativos & Quantidade \\
\hline Desoxicolato de sódio & $1 \%$ \\
\hline Cafeína & $26 \mathrm{mg}$ \\
\hline Silício & $20 \mathrm{mg}$ \\
\hline Lidocaína & $40 \mathrm{mg}$ \\
\hline
\end{tabular}

As aplicações foram realizadas uma vez por semana e a perimetria da circunferência abdominal com fita métrica antes de iniciar o tratamento e ao final das sessões, obtendo assim as medidas obtidas e demonstradas na tabela 3 . A documentação fotográfica foi realizada antes e após a finalização do tratamento.

Tabela 3 - Medidas Inicial e Final da Região Abdominal

\begin{tabular}{|c|c|c|c|}
\hline Região & Medida Inicial $\mathbf{( c m )}$ & Medida Final $\mathbf{( c m )}$ & Observações \\
\hline Abdômen superior & 77 & 70 & Medida aferida 3cm acima do umbigo. \\
\hline Abdômen inferior & 95 & 84 & Medida aferida 3cm abaixo do umbigo. \\
\hline
\end{tabular}

\section{RESULTADOS}

Foi observado que no decorrer das semanas que a paciente teve perda progressiva de circunferência abdominal, resultante ao

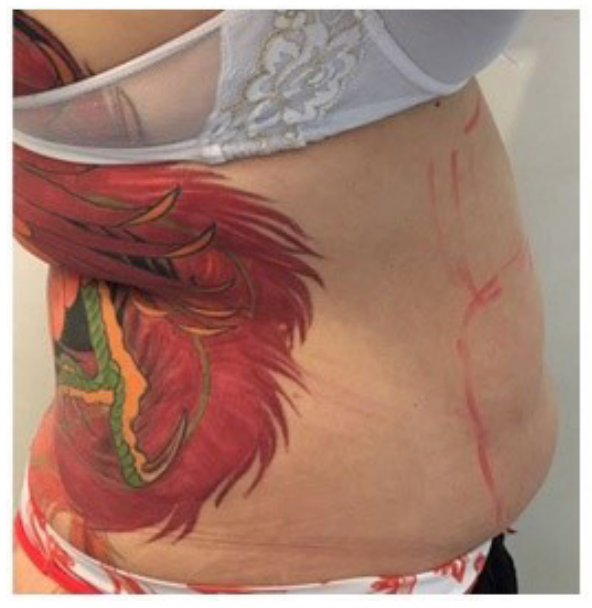

Figura 1: Antes e depois do tratamento aplicado.

\section{DISCUSSÃO}

A aplicação da técnica de carboxiterapia por injeção subcutânea promove aumento da perfusão tecidual, aumento da pressão parcial de oxigênio e redução da circunferência corporal, viabilizando assim, seu efeito lipolítico [5].

No tratamento de carboxiterapia, foi aplicado o gás carbônico final do tratamento na redução de $7 \mathrm{~cm}$ de circunferência em região de abdômen superior e $11 \mathrm{~cm}$ em região de abdômen inferior.

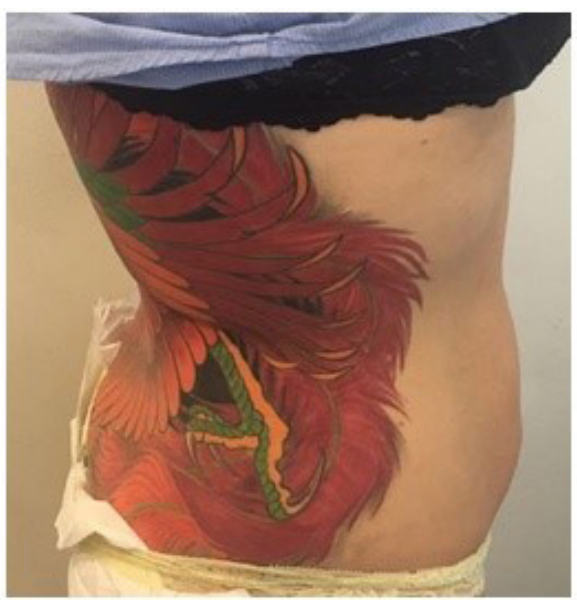

por meio de agulha $30 \mathrm{G}$ no tecido subcutâneo. Por conta da lesão provocada tanto pela agulha quanto pelo gás, o organismo desencadeia um processo inflamatório que visa cicatrizar e reconstruir o tecido lesado. Em virtude do processo de reparação, ocorre angiogênese e fibrogênese [9].

$\mathrm{O}$ ativo Desoxicolato de sódio é apontado na literatura médica como um agente responsável na promoção de lipólise química no tratamento de intradermoterapia. Um estudo demonstrou que, o Desoxicolato de sódio quando injetado por via subcutânea 
promove a lise dos adipócitos e a consequente diminuição do tecido adiposo, perda considerada de leve a moderada [10, 11].

A cafeína estimula o aumento da circulação sanguínea e linfática, pode promover a desagregação das fibroses de gordura, acelerando a hidrólise do excesso dos triglicerídeos [12].

As injeções devem ser realizadas somente na área a ser tratada, a distância entre elas também pode variar entre $1 \mathrm{~cm}$ até $4 \mathrm{~cm}$ entre si. Artigos científicos apontam que as aplicações devem ser realizadas semanalmente ou mensalmente e o número de sessões podem ser entre 4 a 10 durante um tratamento de lipodistrofia localizada[13, 14, 15, 16].

Estudos realizados para verificar a diferença entre a difusão de ativos injetados na derme em $4 \mathrm{~mm}$ até $10 \mathrm{~mm}$ de profundidade, concluiu que nas aplicações realizadas em $10 \mathrm{~mm}$, os ativos difundem-se mais rápido do que aplicações em $4 \mathrm{~mm}$, portanto sugere-se que as injeções sejam realizadas em até $4 \mathrm{~mm}$ de profundidade com intuito de que os ativos permaneçam mais tempo no local injetado [17].

\section{CONCLUSÃO}

De acordo com o resultado encontrado e a visível redução de circunferência abdominal, conclui-se que a associação dos tratamentos de carboxiterapia e intradermoterapia tradicional (mesoterapia) promovem um resultado satisfatório em relação a redução da lipodistrofia localizada.

Os autores sugerem que mais estudos clínicos sejam realizados para demonstrar que a intradermoterapia de forma isolada ou combinada a outras terapias é uma opção segura e eficaz de tratamento eficaz no tratamento da lipodistrofia localizada.

\section{REFERÊNCIAS}

1. SCORZA, Flávia; BORGES, Fábio. Carboxiterapia: uma revisão. Revista Fisioterapia Ser-ano3, №4-2008.

2. SCORZA, Flávio; FIGUEIREDO, Milenna; LIAO, Claudia; BORGES, Fábio. Estudo comparativo dos efeitos da eletrolipólise com uso de TENS modo Burst e modo normal no tratamento de adiposidade localizada abdominal. Ensaios e Ciência: Ciências Biológicas; Agrárias e da Saúde. Vol.XIINº.2, 2008.

3. Cardoso SK, Pereira VCG, Macedo ACB. Efeito imediato da ultracavitação na gordura localizada. Rev. Inspirar Mov. Saúde. 2016; 9(2): 43-49

4. CORRÊA, Michele; GONTIJO, Érika; TONANI, Renata; REIS, Márcia; BORGES, Fábio. Análise da eficácia da carboxiterapia na redução do fibro edema gelóide: estudo piloto. Revista Fisioterapia Ser- ano 3-N 2-2008.

5. BRANDI et al. Carbon dioxide therapy in the treatment of localized adiposities: Clinical study and histopathological correlations. Aesth. Plast. Surg. 170-170. 2001. Dolinsky M.Nutrição funcional. 2.ed. São Paulo, Brasil: Roca; 2009.

6. Pistor M. What is mesotherapy? Chir Dent Fr. 1976;46:59-60.

7. HERREROS, F.O.C.; VELHO, P.E.N.F.; MORAES, A.M.
Mesoterapia: uma revisão bibliográfica. An. Bras. Dermatol., [S.I.], v. 86, n. 1, p. 96-101, 2011.

8. HERREROS FOC, MORAES AM, VELHO PENF. Mesoterapia: uma revisão bibliográfica. An. Bras. Dermatol. 2011;86(1):96-101.

9. SCORZA, F.A.;JAHARA, R. S. Carboxiterapia. São Paulo. Phortes, 2010.

10. ROTUNDA, A.M. et al. Randomized double-blind clinical trial of subcutaneously injected deoxycholate versus a phosphatidylcholine- deoxycholate combination for the reduction of submental fat. Dermatol. Surg., v. 35, n. 1, p. 792-803, 2009.

11. ROTUNDA, A.M.; KOLODNEY, M.S. Mesotherapy and phosphatidylcholine injections: Historical clarification and review. Dermatologic Surgery, v. 32, n. 4, p. 465-480, 2006.

12. MACHADO, A.T.O.M.; NOGUEIRA, A.P.S.; LEÃO, L.T.S.; SANTOS, B.A.; PINHEIRO, L.M.G.; OLIVEIRA, S.S. Benefícios da Massagem Modeladora na Lipodistrofia Localizada. Id online Revista Multidisciplinar e de Psicologia, Maio, vol.11, n.35, p.542553, 2017.

13. TENNSTEDT D., Lachapelle J.M. Effets cutanés indesirables de lamésotherapie. Ann Dermatol Venereol. 1997;124:192-6.

14. MAYA V. Mesotherapy. Indian J Dermatol Venereol Leprol. 2007; 73:60-2.

15. AMIN S.P., Phelps R.G., Goldberg D.J. Mesotherapy for facial skin rejuvenation: a clinical, histological, and electron microscopic evaluation. Dermatol. Surg. 2006; 32:1467-72.

16. JEAN A. Mobilisation des graisses de reserve en mésothérapie. J Med et Chir Derm. 1989; XVI:233-40.

17. MREJEND. Semeiologie, Pharmacocinetique et profondeur des injections en mèsothérapie. In: Bulletin 5 des communications du 6e Congrès International de Mesothérapie; 1992; Bruxelas, Bélgica. Paris: Société Française de Mésothérapie; 1992. p. 13-14. 